\title{
Efficacy and Safety of Corneal Collagen Crosslinking in Treatment of Progressive Keratoconus with Consideration to the Long-Term Results
}

\section{Solodkova EG, Fokin VP, Balalin SV* and Melikhova IA}

S. Fyodorov Eye Microsurgery Federal State Institution, Volgograd Branch, Russia

*Corresponding Author: Balalin SV, S. Fyodorov Eye Microsurgery Federal State Institution, Volgograd Branch, Russia.
Received: May 06, 2021

Published: May 28, 2021

(C) All rights are reserved by Balalin SV., et al.

\section{Abstract}

Purpose: To evaluate the efficacy and safety of a modified technique of corneal collagen crosslinking (CXL) versus standard protocol basing on changes of clinical and morphological characteristics of the cornea in the early and late postoperative period.

Materials and Methods: Two groups of patients were examined: group I (25 persons/25 eyes) consisted of patients treated for progressive keratoconus in accordance with a standard protocol of corneal collagen crosslinking, group II comprised those, who underwent crosslinking, performed in a modified technique - 36 persons (36 eyes). Prior to and after the procedure (1, 3, 6 and 12 months postoperatively), all patients had an extensive eye examination, including optical coherence tomography (OCT) of the cornea (OCT RS-3000, Nidek, Japan), confocal microscopy (Confoscan4, Nidek, Japan).

Results: Faster re-epithelialization of the cornea occurred in the $2^{\text {nd }}$ group. In both groups there was observed a significant increase of UCVA (from $0,15 \pm 0,12$ to $0,35 \pm 0,14$ in the first group and from $0,13 \pm 0,11$ to $0,33 \pm 0,14$ in the second) and BCVA (from $0,3 \pm$ 0,18 to $0,05 \pm 0,12$ in the first group and from $0,4 \pm 0,16$ to $0,6 \pm 0,18$ in the second group) at 6 and 12 months follow-ups. One month postoperatively a well-defined rear stromal haze or "demarcation line" was confirmed by OCT and microscopy in all the treated eyes. By that time the depth of "demarcation line" location averaged $262 \pm 15,0 \mu \mathrm{m}$ in the $1^{\text {st }}$ group and $255 \pm 10,0 \mu \mathrm{m}$ in the $2^{\text {nd }}$. The confocal microscopy revealed that histomorphologic changes were more pronounced in the first group within the whole follow-up period. Starting from the third month of observation a statistically significant downtrend in the rating of all the studied symptoms, with persistence of that tendency in the time that followed - at 6 and 12 months check-ups, was registered in the $2^{\text {nd }}$ group of patients, which pointed at milder and faster corneal recovery after CXL performed in accordance with the modified technique. Endothelial cell density (ECD) remained unchanged in all the cases in both groups within the whole follow-up period.

Conclusion:

1. Positive dynamics in postoperative UCVA and BCVA values, inalterability of ECD in all the cases allowed considering both options of corneal collagen crosslinking safe, with comparable clinical efficacy.

2. However, in view of more evident increase of BCVA in the second group, milder recovery in the early postoperative period and achievement of adequate and safe level of histomorphologic corneal changes after CXL, the modified technique of progressive keratoconus treatment seemed to be more advantageous.

Keywords: Keratoconus; Crosslinking; Modified Technique

Citation: Balalin SV., et al. "Efficacy and Safety of Corneal Collagen Crosslinking in Treatment of Progressive Keratoconus with Consideration to the Long-Term Results". Acta Scientific Ophthalmology 4.6 (2021): 92-100. 


\section{Introduction}

The last decade has been marked by the emergence of new opportunities in treatment of progressive keratoconus $[4,16]$. The most popular method, used at the initial stage of the disease and constituting an alternative to keratoplasty $[5-8,15,18]$, is corneal collagen crosslinking (CXL), proposed in the late 1990s by a group of authors from Dresden University [20-25]. To date, ophthalmic surgeons are searching for possible ways to improve and optimize the classical cross-linking protocol to improve patient tolerability and increase its effectiveness $[3,9,10,14]$.

Since 2014, the Clinic of Volgograd branch of S. Fyodorov Eye Microsurgery Federal State Institution has been applying a modified author's technique of corneal collagen crosslinking, along with the classical protocol [1,2]. The modified technique has been featuring a customized excimer laser de-epithelialization performed to the depth of the epithelial layer basing on the findings of optical coherence tomography (OCT) under the control of intraoperative on-line pachymetry, instead of complete mechanical de-epithelialization. Above that, the modified technique was distinguished by application of a specially designed mesh-like mask with holes of 0.5 $\mathrm{mm}$ in diameter piercing working surface of the instrument, which reduced surgical trauma, accelerated and improved the quality of epithelialization. The above-mentioned treatment modification and a tool for its implementation were registered in the Russian Patent Office as invention N 2602221 of June,18, 2015. Stage of corneal saturation with riboflavin solution (RS) was also amended: a plastic funnel-shaped eyelid retractor was placed circumlimbally on the eyeball surface and filled with $0.5-1.0 \mathrm{ml}$ of $0.1 \%$ riboflavin solution. Thus, the duration of RS soaking was reduced, the quality of saturation was improved and the contact of the de-epithelized cornea with air was eliminated, which diminished severity of corneal syndrome and ameliorated an overall patient's perception of treatment tolerability. That technique was also registered as a patent for invention N 2594434 of June,18, 2015.

This retrospective study estimated safety of classical and modified corneal collagen crosslinking techniques. The main evaluation criteria were the stability of non-corrected and best- corrected visual acuity (UCVA and BCVA), pachymetric, keratotopographic parameters [12,19], endothelial cell density (ECD), as well as the nature and severity of histomorphologic changes in the cornea, assessed by means of confocal microscopy [8].

\section{Purpose of the Study}

To evaluate the effectiveness and safety of the modified corneal collagen crosslinking technique in comparison with conventional surgical protocol basing on the nature of changes in the clinical and morphological characteristics of the cornea in the early and remote postoperative period.

\section{Materials and Methods}

We have followed 61 patients (61 eyes), treated in the Clinic of Volgograd branch of S. Fyodorov Eye Microsurgery Federal State Institution for keratoconus of the I-II degree, among which 25 persons (25 eyes) had a classical corneal collagen crosslinking (I group) and 36 persons (36 eyes) underwent the modified version of CXL (II group). Patients' average age in the I group was 27 years (from 20 to 35 years), 21 male, 4 females. Mean age in the II group was 26 years (from 18 to 55 years), 30 males, 6 females.

Prior to the surgery and in the postoperative period (check-ups at 1, 3, 6, 12 months), all patients underwent an extensive ophthalmological examination, including visometry with assessment of UCVA and BCVA for distance, autorefractometry, ophthalmometry at the apex of keratoconus (Kmax), multipoint pachymetry, optical coherence tomography of the cornea (OCT RS-3000, Nidek, Japan) with epithelial layer thickness evaluation together with calculation of "demarcation line" location depth, ophthalmobiomicroscopy, keratotopographic mapping on Scheimpflug-analyzer "Sirius" (Schwind, Germany) for both the front and the back surface of the cornea, endothelial cell count (ECC), registration of histomorphologic changes of different localization by means of confocal microscopy (Confoscan 4, Nidek, Japan).

In course of the surgery performed in accordance with the modified technique, the dosed de-epithelialization was achieved by excimer laser "Schwind-Amaris" fitted with the option of intraoperative on-line pachymetry (Schwind, Germany). There was used a tool, elaborated in the Clinic of Volgograd branch - a mesh-like mask, which had several removable working surfaces in a form of flat metal plates, whose central zone was punctured with round perforations of $0.5 \pm 0.03 \mathrm{~mm}$ in diameter placed $0,5 \mathrm{~mm}$ apart, acting as an aperture for excimer laser beam in course of corneal de-epithelialization. The working part of the tool was mounted at a distance of 2 - $3 \mathrm{~mm}$ from the surface of the epithelium in order not to cause any mechanical impact on the cornea. The excimer laser

Citation: Balalin SV., et al. "Efficacy and Safety of Corneal Collagen Crosslinking in Treatment of Progressive Keratoconus with Consideration to the Long-Term Results". Acta Scientific Ophthalmology 4.6 (2021): 92-100. 
exposure was adjusted in a way not to exceed the depth of epithelial layer which allowed obtaining precise scarification under all the perforations (Figure 1). Ultraviolet irradiation was performed by means of "UV-X" (version 1000) appliance (IROC, Switzerland). Silicone hydrogel soft contact lenses were prescribed to all patients for the duration of re-epithelialization. Topical medication in the early postoperative period in both groups under study consisted of $0.3 \%$ tobramycin solution instilled in regimen of 1 drop 4 times a day for a week. A complete re-epithelialization of the cornea being achieved, $0.1 \%$ dexamethasone solution was prescribed 1 drop 3 times a day for three weeks.

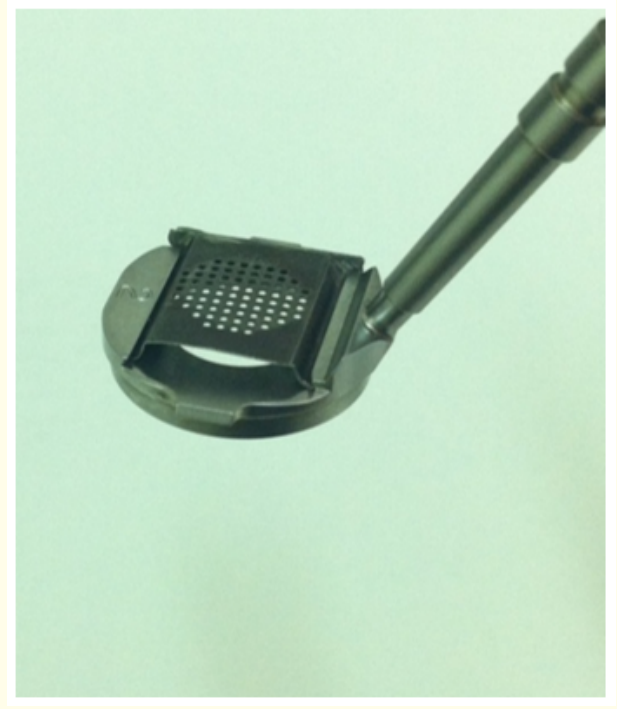

Figure 1: Mask for dosed excimer laser de-epithelialization.

The effectiveness of the methods was assessed by stabilization and improvement of functional parameters, as well as by the nature and severity of characteristic histomorphologic changes in the cornea as a result of corneal collagen crosslinking. Safety was estimated basing on the level of various complications, both in the early and in the distant postoperative period.

\section{Results}

The duration of corneal saturation with riboflavin solution averaged $30.2 \pm 0.5 \mathrm{~min}$ in the classical protocol and $22.1 \pm 0.5 \mathrm{~min}$ in the modified protocol. Saturation time shortening resulted from pressure caused by liquid delivered through the funnel after par- tial de-epithelialization of the cornea. That stage of the surgery was better tolerated by patients of the second group. The demand in additional instillations of anesthetic solution in course of procedures, performed in compliance with the modified technique was 3.7 times lower in comparison to the standard CXL. Thus, number of additional instillations of anesthetic was $4.5 \pm 0.1$ times in the first group and only $1.2 \pm 0.1$ times in the second $(\mathrm{t}=23.5 ; \mathrm{p}<$ $0.001)$. That difference was due to elimination of air-de-epithelized cornea contact in cases when a funnel-shaped eyelid retractor was being used, which ultimately reduced the severity of the corneal syndrome.

The time, needed for complete corneal epithelialization, was $84.1 \pm 1.2$ hours in the first group and $24.2 \pm 1.0$ hours in the second (Figure $2 \mathrm{a}$ and $2 \mathrm{~b}$ ). The difference between the average values was statistically significant $(t=23.1 ; p<0.001)$. The results indicated that the modified technique was less traumatic.

The difference between the mean values of UCVA in both groups was statistically significant for the follow-up period of six months and one year $(\mathrm{t} \geq 2 ; \mathrm{p}<0.05)$. The difference between the mean values of BCVA in both groups was also statistically relevant for the follow-up period of 6 and 12 months ( $\mathrm{t}>2, \mathrm{p}<0.01$ ) (Table 1 and 2).

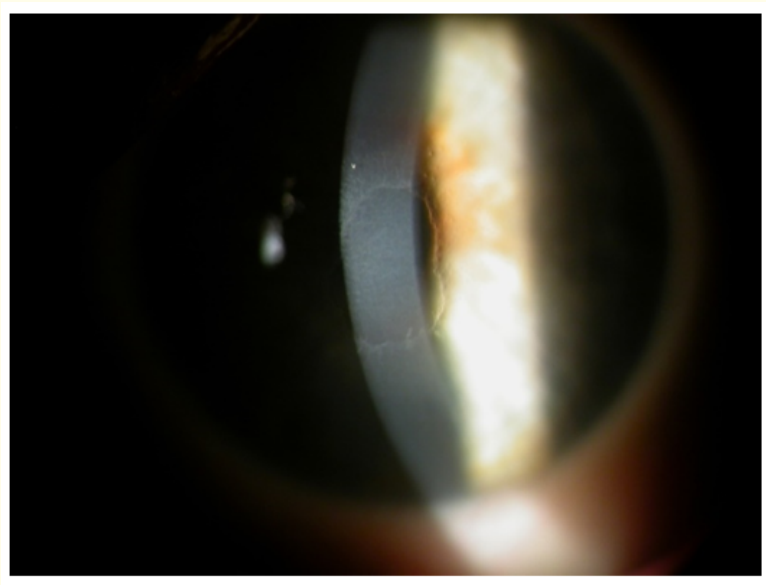

Figure 2a: Epithelial erosion on the $3^{\text {rd }}$ day after surgery, $1^{\text {st }}$ group. 


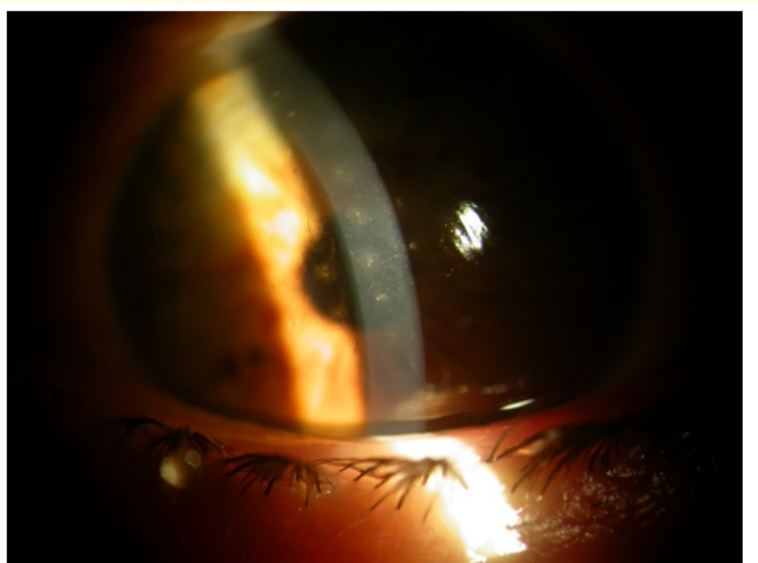

Figure 2b: Complete re-epithelialization, 1 day after surgery, $2^{\text {nd }}$ group.
It was noted that one month postoperatively the Kmax value had slightly increased in reference to the initial level in the center of the cornea and at the apex of keratoconus as well in both groups. In the months that followed, the Kmax value at these locations gradually decreased. One year postoperatively, that decrease was 5,3\% in the central optical zone, $3,0 \%$ at the apex of keratoconus in the $1^{\text {st }}$ group and $5,7 \%$ in the center and $4,7 \%$ at the apex of keratoconus in the $2^{\text {nd }}$ group. The difference in that parameter was statistically significant for the follow-up period of 1 year in both groups $(\mathrm{t}=2.3$, $\mathrm{p}<0.05$ ). Gradual downtrend in ophthalmometric values, in our opinion, was associated with corneal stromal compaction arising and progressing with time as a result of CXL. UCVA improvement in both groups correlated with decline of ophthalmometric findings obtained in the central optical zone (Table 1 and 2).

There was revealed a drastic decrease in pachymetric values within the follow-up period from 1 to 6 months in both groups

\begin{tabular}{|l|c|c|c|c|c|}
\hline \multicolumn{1}{|c|}{ Parameter } & Pre0p & 1 month & 3 months & $\mathbf{6}$ months & 12 months \\
\hline UCVA & $0,15 \pm 0,12^{*}$ & $0,125 \pm 0,07$ & $0,21 \pm 0,18$ & $0,26 \pm 0,09^{* *}$ & $0,35 \pm 0,14^{* *}$ \\
\hline BCVA & $0,3 \pm 0,18^{*}$ & $0,36 \pm 0,19$ & $0,45 \pm 0,11^{* *}$ & $0,48 \pm 0,12^{* *}$ & $0,5 \pm 0,12^{* *}$ \\
\hline K center & $47,0 \pm 2,6^{*}$ & $48,6 \pm 1,5^{* *}$ & $46,0 \pm 1,5^{* *}$ & $45,4 \pm 1,7^{* *}$ & $44,5 \pm 2,3^{* *}$ \\
\hline K apex & $58,3 \pm 4,3^{*}$ & $59,9 \pm 2,1^{* *}$ & $58,7 \pm 2,9$ & $58,5 \pm 2,5$ & $56,5 \pm 1,8^{* *}$ \\
\hline Pachymetry center & $456,0 \pm 14,6^{*}$ & $420,0 \pm 13,6^{* *}$ & $420,0 \pm 14,7^{* *}$ & $412,0 \pm 9,7^{* *}$ & $420,0 \pm 8,0^{* *}$ \\
\hline Pachymetry apex & $440,0 \pm 16,3^{*}$ & $394,0 \pm 12,0^{* *}$ & $391,0 \pm 11,3^{* *}$ & $391,0 \pm 9,2^{* *}$ & $395,0 \pm 7,0^{* *}$ \\
\hline ECD & $2669,0 \pm 80^{*}$ & $2651,0 \pm 85^{*}$ & $2686,0 \pm 80^{*}$ & $2763,0 \pm 70^{*}$ & $2640,0 \pm 70^{*}$ \\
\hline
\end{tabular}

Table 1: The average values of NCVA, BCVA, findings of pachymetry and confocal microscopy of the cornea in patients of the I group in course of dynamic observation, $\mathrm{M} \pm \sigma$.

The differences between the mean values marked with * and ${ }^{* *}$ are statistically significant $(\mathrm{p}<0.05)$.

\begin{tabular}{|l|c|c|c|c|c|}
\hline \multicolumn{1}{|c|}{ Parameter } & Pre0p & 1 month & 3 months & $\mathbf{6}$ months & 12 months \\
\hline UCVA & $0,13 \pm 0,11^{*}$ & $0,18 \pm 0,16$ & $0,19 \pm 0,11^{* *}$ & $0,25 \pm 0,12^{* *}$ & $0,33 \pm 0,14^{* *}$ \\
\hline BCVA & $0,4 \pm 0,16^{*}$ & $0,4 \pm 0,15$ & $0,45 \pm 0,9$ & $0,5 \pm 0,10^{* *}$ & $0,67 \pm 0,18^{* *}$ \\
\hline K center & $48,8 \pm 2,1^{*}$ & $49,3 \pm 2,5$ & $47,7 \pm 2,3$ & $46,5 \pm 1,8^{* *}$ & $46,0 \pm 2,8^{* *}$ \\
\hline K apex & $57,2 \pm 3,9^{*}$ & $57,7 \pm 2,1$ & $57,0 \pm 1,7$ & $56,0 \pm 1,9$ & $54,5 \pm 1,5^{* *}$ \\
\hline Pachymetry center & $473,0 \pm 15,2^{*}$ & $450,0 \pm 15,7^{* *}$ & $451,0 \pm 15,6^{* *}$ & $457,0 \pm 16,4^{* *}$ & $458,0 \pm 15,2^{* *}$ \\
\hline Pachymetry apex & $458,0 \pm 14,0^{*}$ & $425,0 \pm 14,4^{* *}$ & $420,0 \pm 14,0^{* *}$ & $419,0 \pm 14,0^{* *}$ & $424,0 \pm 15,7^{* *}$ \\
\hline ECD & $2541 \pm 35^{*}$ & $2523 \pm 35^{*}$ & $2530 \pm 40^{*}$ & $2519 \pm 35^{*}$ & $2543 \pm 40^{*}$ \\
\hline
\end{tabular}

Table 2: The average values of UCVA, BCVA, findings of pachymetry and confocal microscopy of the cornea in patients of the II group in course of dynamic observation, $\mathrm{M} \pm \sigma$.

The differences between the mean values marked with * and ${ }^{* *}$ are statistically significant $(\mathrm{p}<0.05)$.

Citation: Balalin SV., et al. "Efficacy and Safety of Corneal Collagen Crosslinking in Treatment of Progressive Keratoconus with Consideration to the Long-Term Results". Acta Scientific Ophthalmology 4.6 (2021): 92-100. 
(more pronounced in the $1^{\text {st }}$ group). Check-up, performed six months postoperatively, showed tendency to stabilization of pachymetric values. At examination, made one year after the surgery, positive dynamics was observed. The difference turned out to be statistically significant at all periods of observation in the studied groups ( $\mathrm{t} \geq 2.0 ; \mathrm{p}<0.05)$. In the first group, the residual corneal thickness obtained after mechanical de-epithelialization mainly averaged $400 \mu \mathrm{m}$ required for a safe ultraviolet irradiation. In cases when corneal thickness was less than $400 \mu \mathrm{m}$, cornea was irrigated with BSS until a secure thickness value was being achieved.

The downtrend in corneal thickness values, observed within the follow-up period, may also be associated with gradual stromal compaction (Table 1 and 2). The initial ECD exceeded 2600/ $\mathrm{mm}^{2}$ in the $1^{\text {st }}$ group and $2500 / \mathrm{mm}^{2}$ in the $2^{\text {nd }}$. Not a single case of treatment-induced alteration of that parameter was registered within the follow-up period.

One month postoperatively, a gentle linear opacification - the so-called "demarcation line" or "posterior stromal haze" appeared in the middle and posterior stromal layers, which was confirmed by OCT and biomicroscopy in all the cases (Figure $3 \mathrm{a}$ and $3 \mathrm{~b}$ ). At that time, the "demarcation line" was located at the depth of 262,0 $\pm 15,0 \mu \mathrm{m}$ in the $1^{\text {st }}$ group of patients and at the depth of 255,0 $\pm 10,0 \mu \mathrm{m}$ in the $2^{\text {nd }}$ group. Subsequent OCT imaging, performed at check-ups of 3, 6, 12 months postop, revealed that depth of demarcation line location had gradually decreased, i.e. "stromal haze" had moved towards the front layers. For instance, three months postop depth of location in the $1^{\text {st }}$ and $2^{\text {nd }}$ group was $249,0 \pm 12,0$ and $240,0 \pm 10,0 \mu \mathrm{m}$, six months postop $-229,0 \pm 10,0$ and 220,0 $\pm 10,0 \mu \mathrm{m}$, twelve months postop $-217,0 \pm 10,0$ and $205,0 \pm 10,0$ $\mu \mathrm{m}$, respectively (Table 3 ). Our results were consistent with the literature $[11,13]$.

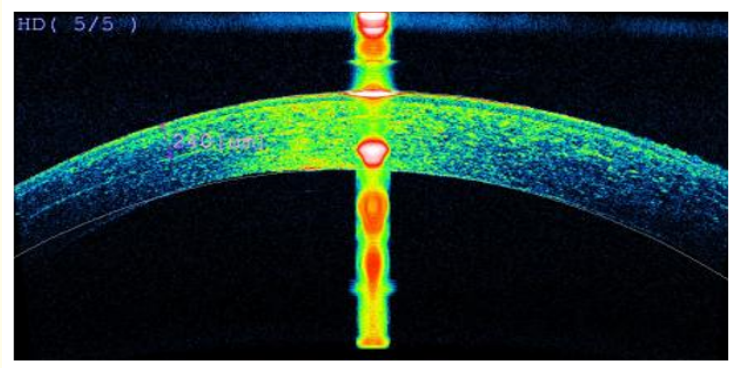

$3 a$

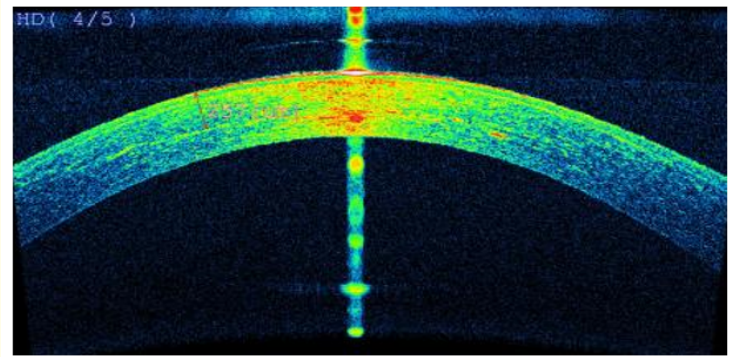

$3 \mathrm{~b}$

Figure 3: Demarcation line, 1 month postoperatively, $\mathrm{a}-1^{\text {st }}$ group, $\mathrm{b}-2^{\text {nd }}$ group.

Histomorphologic changes detected by confocal microscopy - corneal edema in a form of ECM transparency decrease, epitheliopathy, activation of stromal keratocytes, as well as fibroplasia phenomenon, were assessed by means of a specially developed 4-point scale. Within the first two weeks following the surgery, the biomicroscopic study has revealed epithelial and stromal edema in all the cases, which had completely resolved under instillations of corticosteroids. The severity of stromal edema was characterized as follows: 0 points - a slight decrease of ECM transparency, 1 point

\begin{tabular}{|l|c|c|c|c|}
\hline Patients under study & 1 month & 3 months & 6 months & 12 months \\
\hline $1^{\text {st }}$ group & $262,0 \pm 15,0^{*}$ & $249,0 \pm 12,0^{*}$ & $229,0 \pm 10,0^{*}$ & $217,0 \pm 10,0^{*}$ \\
\hline $2^{\text {nd }}$ group & $255,0 \pm 10,0^{*}$ & $240,0 \pm 10,0^{*}$ & $220,0 \pm 10,0^{*}$ & $205,0 \pm 10,0^{*}$ \\
\hline
\end{tabular}

Table 3: The average depth of demarcation line location, $\mathrm{M} \pm \sigma$

Difference between the mean values, marked with *, was not statistically significant $(t>2,0 ; p<0,05)$.

- a moderately expressed deviance in ECM transparency involving epithelium and anterior stroma (propagation depth not exceeding
$100 \mu \mathrm{m}), 2$ points - a pronounced decrease of ECM transparency within the epithelial layer and corneal stroma, extending to the

Citation: Balalin SV., et al. "Efficacy and Safety of Corneal Collagen Crosslinking in Treatment of Progressive Keratoconus with Consideration to the Long-Term Results". Acta Scientific Ophthalmology 4.6 (2021): 92-100. 
depth from 100 to $300 \mu \mathrm{m}, 3$ points - an apparent decline of ECM transparency, outspreading downwards $300 \mu \mathrm{m}$.

The state of the epithelial layer was evaluated by severity of epitheliopathy both within the basal layer and the layer of polyhedral (wing) cells: 0 points - no change, 1 point - a feeble pseudo-keratinization of wing cells (number of pseudo-keratinized epitheliocytes not exceeding 10 in the field of view $(0,2 \times 0,15 \mathrm{~mm}), 2$ points - a marked pseudo-keratinization of wing cells (counting from 10 to 30 in the field of view), up to 30 epitheliocytes with abnormal hyper-reflective nuclei within the basal layer, 3 points - more than 30 pseudo-keratinized epitheliocytes in the layer of wing cells, more than 30 epitheliocytes with hyper-reflective nuclei in the basal layer (Figure $4 \mathrm{a}$ and $4 \mathrm{~b}$ ).

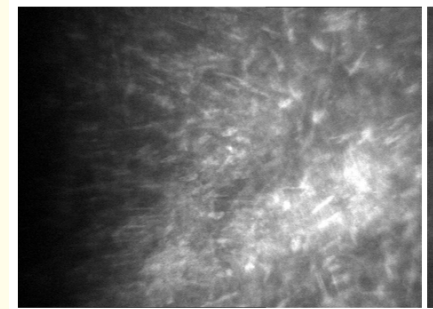

$4 \mathrm{a}$

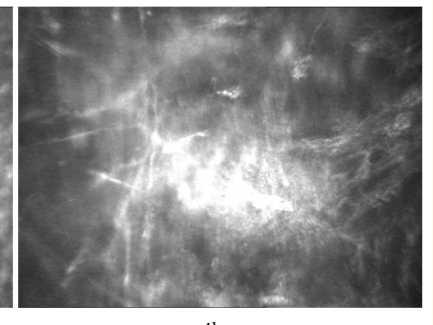

$4 \mathrm{~b}$
Figure 4: Fibroplasia within stromal layer, the depth corresponds to demarcation line, at 1 month check-up, $\mathrm{a}-1^{\text {st }}$ group, b-2 ${ }^{\text {nd }}$ group, 1 point.

Confocal microscopy also helped to estimate changes in stromal cell structure basing on the number of active keratocytes with bright, hyper-reflective nuclei: 0 points - no active keratocytes, 1 point - active keratocytes in the field of view (counting less than 10), 2 points - number of active keratocytes in a range from 10 to 30,3 points - number of active keratocytes exceeded 30 .

One month postoperatively, a linear opacification, confined in middle and posterior stromal layers - the so-called "posterior stromal haze" was observed in all the cases (Figure $5 \mathrm{a}$ and $5 \mathrm{~b}$ ). The intensity of fibroplastic changes was also assessed by means of a 4-point scale: 0 points - transparent cornea, 1 point - focal opacities, 2 points - diffuse soft stromal haze that did not affect visual acuity, 3 points - diffuse moderate stromal haze that aggravated UCVA and BCVA [17] (Table 4). The RF patent for invention $\mathrm{N}$ 2578821 of 03.03 .2015 was granted for the-above mentioned method of postsurgical corneal state evaluation.
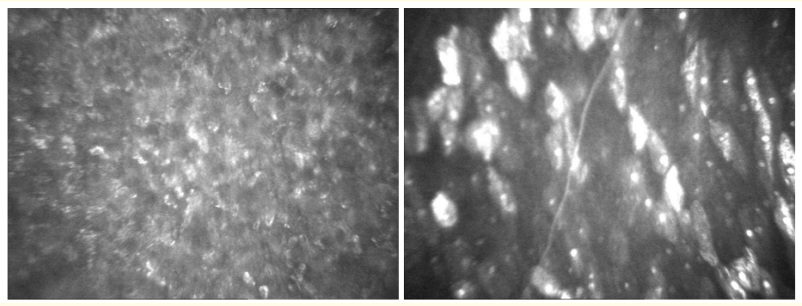

Figure 5a: Epitheliopathy within the basal and superficial layer of the epithelium, 1 month postoperatively, $1^{\text {st }}$ group, 3 points.
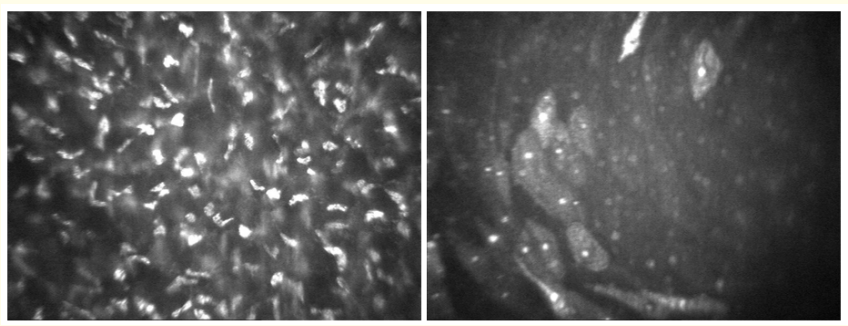

Figure 5b: Epitheliopathy of the basal and superficial layer of the epithelium, 1 month postoperatively, $2^{\text {nd }}$ group, 2 points.

ECD remained unchanged through all the follow-up period.

The data, displayed in the table 4, demonstrated that the described histomorphologic changes were more pronounced in the $1^{\text {st }}$ group through all the follow-up period. Starting from the third month of observation a statistically significant downtrend in the rating of all the studied symptoms, with persistence of that tendency in the time that followed - at 6 and 12 months check-ups, was registered in the $2^{\text {nd }}$ group of patients, which pointed at milder and faster corneal recovery after CXL performed in accordance with the modified technique. 


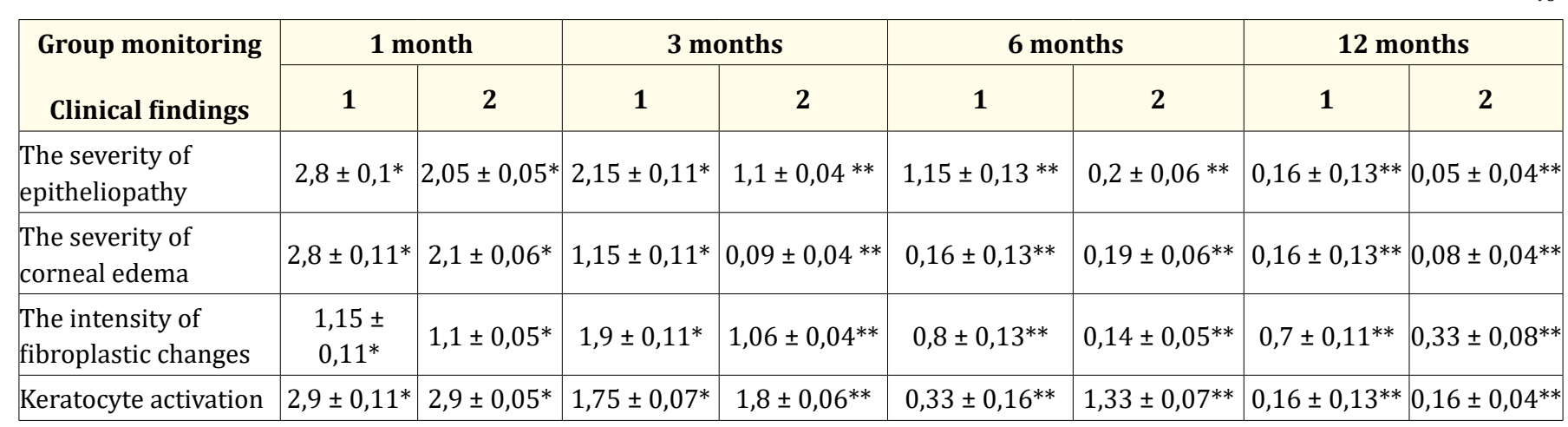

Table 4: Evaluation of histomorphologic changes in the cornea after CXL, expressed in points, at different follow-up periods in the studied groups of patients, $\mathrm{M} \pm \sigma$.

The differences between the mean values marked with * and ${ }^{* *}$ were statistically significant $(\mathrm{p}<0.05)$.

Both standard and modified techniques induced the following complications: non-infectious keratitis and reactive iridocyclitis 1 case in each group $(0.25 \%$ and $0.36 \%$, respectively). There was registered one case of prolonged re-epithelialization, with ensuing rough fibroplastic changes requiring long-term treatment, in the $1^{\text {st }}$ group of patients $(0.25 \%)$. No cases of endothelial cell damage, ECD decrease, lens opacities, macular edema were revealed.

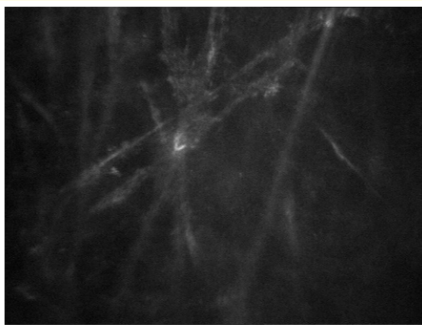

6a

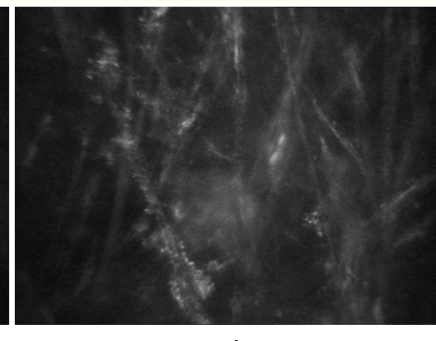

$6 b$
Figure 6: Polymorphic collagen fibers in the anterior stroma, observation period 12 months, a- $1^{\text {st }}$ group, $b-2^{\text {nd }}$ group.

\section{Discussion}

The duration of the corneal saturation with riboflavin solution was shortened owing to the pressure caused by liquid delivered through the funnel after partial de-epithelialization of the cornea. That stage of the surgery was better tolerated by patients of the second group, which was confirmed by lesser demand in additional

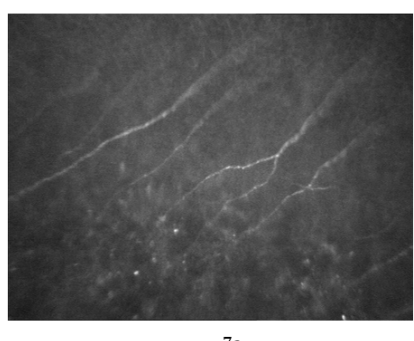

$7 a$

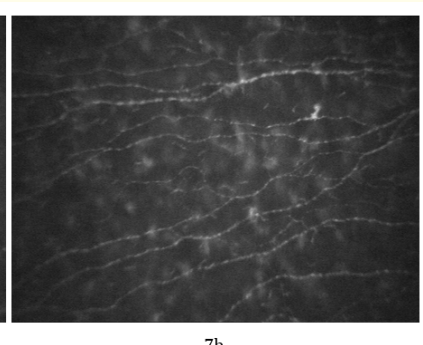

$7 \mathrm{~b}$
Figure 7: Subepithelial reinnervation, follow-up period of 6 months, a- $1^{\text {st }}$ group, $b-2^{\text {nd }}$ group.

instillations of anesthetic solution in course of procedures. That difference was due to elimination of air-de-epithelized cornea contact in cases when a funnel-shaped eyelid retractor was being used, which ultimately reduced the severity of the corneal syndrome.

A gradual downtrend in ophthalmometric values, in our opinion, was associated with corneal stromal compaction arising and progressing with time as a result of CXL. UCVA improvement in both groups correlated with decline of ophthalmometric findings obtained in the central optical zone, which was supported by the results of previous studies. For example, G. Wollensak (2003) analyzed long-term results of CXL in his work [23]. Postoperative examination was spaced at 6 months intervals and included visom- 
etry, refractometry, ECD evaluation. Basing on data from 31 cases the author had registered a decline in SE by average of 2,87 diopters and neither damage of endothelial cells, nor ECD decrease.

The downtrend in corneal thickness values, observed within the follow-up period, may also be associated with gradual stromal compaction and demarcation line shift towards the front layers. The obtained results were consistent with the literature [11,13].

Histomorphologic changes detected by confocal microscopy - corneal edema in a form of ECM transparency decrease, epitheliopathy, activation of stromal keratocytes, as well as fibroplasia phenomenon were noted in all the cases [17]. The data, summarized in the table 4, demonstrated that the described histomorphologic changes were more pronounced in the $1^{\text {st }}$ group through all the follow-up period. Starting from the third month of observation a statistically significant downtrend in the rating of all the studied symptoms, with persistence of that tendency in the time that followed - at 6 and 12 months check-ups, was registered in the $2^{\text {nd }}$ group of patients, which pointed at milder and faster corneal recovery after CXL performed in accordance with the modified technique.

\section{Conclusion}

Positive dynamics in postoperative UCVA and BCVA values, inalterability of ECD in all the cases allowed considering both options of corneal collagen crosslinking safe, with comparable clinical efficacy.

However, in view of more expressed increase of BCVA in the second group, milder recovery in the early postoperative period and achievement of adequate and safe level of histomorphologic corneal changes after CXL, the modified technique of progressive keratoconus treatment seems to be more advantageous.

\section{Bibliography}

1. Solodkova EG and Remesnikov IA. "Analysis of long-term results of corneal collagen crosslinking in the treatment of progressive keratoconus". The Journal of Practical Medicine 1 (2021): 118-120.

2. Solodkova EG., et al. "Comparative analysis of methods for keratoconus treatment. Abstracts of The VI Annual All-Russian Scientific Practical Conference of Young Scientists "Cur- rent Problems of Ophthalmology" within the Scientific Practical Conference "Fyodorov Memorial Lectures-2011". Moscow (2011): 229-231.

3. Pashtaev NP and Zotov VV. "A new method of corneal collagen crosslinking in the treatment of patients with keratoconus. Preliminary results". Abstracts The Scientific Practical Conference "Fyodorov Memorial Lectures" (2011): 84.

4. Feinbaum C. "Current Aspects of Keratoconus Etiopathogenesis and Treatment". Ophthalmosurgery 3 (2011): 80-83.

5. Anwar M and Teichmann KD. "Deep lamellar keratoplasty: Surgical techniques for anterior lamellar keratoplasty with and without baring of Descemet's membrane". Cornea 21 (2002): 374-383.

6. Bahar I., et al. "Comparison of three different techniques of corneal transplantation for keratoconus". International Ophthalmology 28 (2008): 141-146.

7. Colin J., et al. "Correcting keratoconus with intracorneal rings". The Journal of Cataract and Refractive Surgery 26 (2000): 1117-1122.

8. Efron N and Hollingsworth J. "New perspectives on keratoconus as revealed by corneal confocal microscopy". Clinical and Experimental Optometry 91.1 (2008): 34-35.

9. Ferrara de A and Cunha P. "Tecnica cirurgica para correcao de myopia Anel Corneano Intra-Estromal". Revista Brasileira de Oftalmologia 54 (1995): 577-588.

10. Kaya V., et al. "Efficacy of Corneal Collagen Cross-linking Using a Custom Epithelial Debridement Technique in Thin Corneas: A confocal Microscopy Study". Journal of Refractive Surgery 27.6 (2011): 444-450.

11. Li Y., et al. "Keratoconus diagnosis with optical coherence tomography pachymetry mapping". Ophthalmology 115.12 (2008): 2159-2166.

12. Maeda N., et al. "Automated keratoconus screening with corneal topography analysis". Investigative Ophthalmology and Visual Science 35 (1994): 2749-2757. 
13. Mazzotta C., et al. "Stromal haze after combined riboflavinUVA corneal collagen cross-linking in keratoconus: in vivo confocal microscopic evaluation". Clinical and Experimental Ophthalmology 35 (2007): 580-582.

14. Pinelli R. "C3-R treatment opens new frontiers for keratoconus and corneal ectasia". Eyeworld 34 (2007): 36-39.

15. Price FW and Price MO. "Adult keratoplasty: has the prognosis improved in the last 25 years?" International Ophthalmology 28.3 (2008): 41-146.

16. Rabinowitz YS. "Keratoconus". Survey of Ophthalmology 42 (1998): 297-319.

17. Raiskup F., et al. "Permanent corneal haze after riboflavinUVA-induced cross-linking in keratoconus". Journal of Refractive Surgery 25 (2009): 824-828.

18. Roe RH., et al. "The value-based medicine comparative effectiveness and cost-effectiveness of penetrating keratoplasty for keratoconus". Cornea 27.9 (2008): 1001-1007.

19. Smolek MK and Klyce SD. "Neural network classification of corneal topography". Investigative Ophthalmology and Visual Science 38 (1997): 2290-2299.

20. Spoerl E., et al. "Crosslinking Effects in the cornea of Rabbits". Ophthalmology 97 (2000): 203-206.

21. Spoerl E., et al. "Thermomechanical Behavior of CollagenCrosslinked Porcine Cornea". Ophthalmologica 218 (2004): 136-140.

22. Spoerl E., et al. "Increased Resistance of Crosslinked Cornea against Enzymatic Digestion". Current Eye Research 29.1 (2004): 35-40.

23. Wollensak G., et al. "Riboflavin/Ultraviolet-A Induced Collagen- Crosslinking for the Treatment of Keratoconus". American Journal of Ophthalmology 135 (2003): 620-627.

24. Wollensak G., et al. "Collagen Fiber Diameter in the Rabbit Cornea after Collagen-Crosslinking by Riboflavin/UVA". Journal Cornea 23 (2004): 503-507.
25. Wollensak G., et al. "Collagen Fiber Diameter in the Rabbit Cornea after Collagen- Crosslinking by Riboflavin/UVA". Journal Cornea 23.5 (2004): 503-507.

\section{Volume 4 Issue 6 June 2021 \\ (C) All rights are reserved by Balalin SV., et al.}

